\title{
The Rise of Telemedicine: Lessons from a Global Pandemic
}

\author{
Authors: \\ Samantha Seivert, Melissa E. Badowski \\ 1. College of Pharmacy, University of Illinois at Chicago, Chicago, Illinois, USA \\ 2. Infectious Diseases Pharmacotherapy, Department of Pharmacy Practice, College \\ of Pharmacy, University of Illinois at Chicago, Chicago, Illinois, USA \\ *Correspondence to badowski@uic.edu
}

Disclosure: $\quad$ The authors have declared no conflicts of interest.

Received: $\quad 30.09 .20$

Accepted: $\quad 14.12 .20$

Keywords: $\quad$ Coronavirus disease (COVID-19), pandemic, severe acute respiratory syndrome coronavirus 2 (SARS-CoV-2), telehealth, telemedicine.

Citation:

EMJ Innov. 2020;5[1]:64-69.

\section{Abstract}

Telemedicine has been available for healthcare systems to assist patient care for many years; however, it was not until recently that the field of telemedicine exploded. Inconsistent coverage of telemedicine services as well as a general level of unfamiliarity with the technology required to perform telemedicine services contributed to the lack of its widespread use. The coronavirus disease (COVID-19) pandemic drove the institution of telemedicine in all areas of healthcare. Healthcare institutions around the world adapted both inpatient and outpatient services in order to utilise telemedicine. The implementation of telemedicine can partly be attributed to the expansion of insurance coverage as well as the relaxation of technology requirements to avoid Health Insurance Portability and Accountability Act (HIPAA) violations. During the global pandemic, telemedicine helped to preserve personal protective equipment during a worldwide shortage, protect healthcare workers from being infected, and allowed the monitoring of patients' chronic conditions without putting them at risk by attending medical settings. The COVID-19 outbreak has highlighted the advantages that telemedicine has to offer and has served as the push many health systems needed to implement telemedicine services more widely across these institutions. This article highlights the role of telemedicine during the ongoing COVID-19 global pandemic.

\section{INTRODUCTION}

Telemedicine has developed how healthcare practitioners interact and provide medical care to patients. Overall, telehealth includes a variety of tools and technology resources that effectively deliver care to a patient. Telemedicine is a subset of telehealth which refers to a direct interaction between a healthcare provider and the patient. Telemedicine can be useful in providing follow-up that require frequent monitoring. ${ }^{1}$ Aside from the outpatient setting, telemedicine has also proved effective for monitoring patients in an intensive care setting. ${ }^{2}$ Telemedicine has a multitude of benefits for the patient, the healthcare system, and the provider (Table 1),1,3 With the various benefits available to each participating party, it is clear that the use of telemedicine could completely change the way medicine is practised. 
Table 1: Advantages of telemedicine for patients, providers, and healthcare systems.

\begin{tabular}{|l|l|l|}
\hline Patient & Provider & Healthcare system \\
\hline $\begin{array}{l}\text { Increased continuity of care leading to } \\
\text { better patient outcomes }\end{array}$ & Capability to work from anywhere & $\begin{array}{l}\text { Ability to provide care to underserved } \\
\text { populations/areas of the community }\end{array}$ \\
\hline $\begin{array}{l}\text { Access to medical professionals } \\
\text { outside of typical clinic hours }\end{array}$ & Reduced commute time & $\begin{array}{l}\text { Possibility to expand clinical services } \\
\text { even when space does not permit } \\
\text { expansion }\end{array}$ \\
\cline { 1 - 2 } $\begin{array}{l}\text { Reduced travel burden } \\
\text { Cost savings (vehicle parking, loss of } \\
\text { wages from time off work) }\end{array}$ & $\begin{array}{l}\text { Increase in job satisfaction with the } \\
\text { implementation of telecommuting } \\
\text { commute long provide care to rural areas }\end{array}$ & $\begin{array}{l}\text { Reduction in clinic congestion } \\
\text { with additional support from remote- } \\
\text { staff }\end{array}$ \\
\cline { 1 - 2 } $\begin{array}{l}\text { More likely to return for follow-up } \\
\text { visits when it is convenient for } \\
\text { the patient }\end{array}$ & & \\
\hline
\end{tabular}

Adapted from American Medical Association (AMA), ${ }^{1}$ Barnett et al., ${ }^{3}$ and Martin et al. ${ }^{4}$

\section{PAST}

Telemedicine has been available to clinicians for many years; however, it was not until recently that telemedicine has truly been embraced. According to data from OptumLabs Data Warehouse ${ }^{3}$ (Optum, Eden Prairie, Minnesota, USA), the use of telemedicine has increased between 2005 and 2017; however, overall use was still low and use within the health system was infrequent. ${ }^{3}$ While the use of telemedicine for mental health services grew steadily between 2016 and 2017, its use for other specialties, or even primary care, was still not universally implemented. ${ }^{3}$ A survey conducted by the American Hospital Association (AHA) reported the use of telehealth within the hospital system increased from 35\% in 2010 to $76 \%$ in 2017 . As of $2017,61.2 \%$ of hospitals in the USA used telehealth for remote patient monitoring. ${ }^{5}$ Telemedicine was seen by many as a last resort to treat their patients rather than an equally effective alternative to face-to-face patient care. ${ }^{6}$

Prior to the coronavirus disease (COVID-19) pandemic, many barriers to the widespread implementation and use of telemedicine existed. Logistics of training staff on the use of a new electronic platform for telemedicine was seen as a considerable obstacle to implementing its use across healthcare systems. In addition to the lack of desire to train staff on a new skill set, there was also a general unwillingness from clinicians to convert their current practices and learn a new way of providing medical care since the current mode of practice had been working sufficiently for many years. ${ }^{7,8}$

Prior to 2016, the European Union (EU) lacked synchronised regulations regarding the use of telemedicine across European countries, which limited its development. ${ }^{9}$ Both patients and physicians became comfortable with the way things were and showed little interest in making drastic changes. ${ }^{10}$ Many healthcare professionals were not familiar with the endless technological possibilities that then became available to facilitate patient care via telemedicine. The lack of consistent reimbursement for telemedicine services also discouraged many health systems from implementing telemedicine on a wider scale. ${ }^{10}$ Another prevalent barrier to implementation of telemedicine was the misconception that it was only beneficial to help patients living in rural areas, leaving healthcare providers and systems without the sense of urgency to change current practices. ${ }^{8}$ 


\section{PRESENT}

The COVID-19 pandemic forced rapid implementation of telemedicine into everyday practice. What once seemed like a lofty, futuristic goal became reality within the blink of an eye as various levels of telemedicine were globally transformed into practice. ${ }^{10}$ The large-scale conversion to telemedicine visits over in-person visits was fueled by fear of the unknown as health systems entered the heart of a global pandemic. Firstly, the use of telemedicine in nonurgent cases would ensure continuity of care while patients and healthcare staff were able to remain socially distant. In addition, the conversion to telemedicine also helped to reduce the risk of exposure to COVID-19 for healthcare workers thanks to less contact with potentially infected patients. Exposure and infection of healthcare workers had the potential to place a serious strain on the rest of healthcare staff and essential resources. An employee who had been exposed to an infected patient would be required to quarantine meaning one less healthcare professional able to work with the increased patient load. ${ }^{7}$ In addition, not only would the employee need to quarantine, early on in the spread of COVID-19 there was no consistent data on how long self-quarantine should last. Finally, healthcare societies began publishing more guidelines on ways to properly implement telemedicine while still maintaining optimal patient care. ${ }^{11}$ In Western China, the telemedicine infrastructure was used to educate healthcare workers about COVID-19. Information on methods to standardise the diagnosis of COVID-19, approaches to control the spread of COVID-19 through the hospital, and ways to effectively protect hospital workers were provided to healthcare workers across the country. ${ }^{12}$ Telemedicine had never before been implemented on such a wide scale and each day brought new challenges and strategies to overcome the obstacles to provide optimal patient care.

Hospital workflow changed drastically as COVID-19 spread across the world. Many changes were implemented to preserve personal protective equipment (PPE) during the global shortage. The use of telemedicine for nonurgent visits allowed PPE to be reserved in more urgent patient situations which required on-site management. At Baylor Scott \& White
All Saints Medical Center (Fort Worth, Texas, USA), the emergency department began using telemedicine communication techniques so that physicians could communicate with patients who tested positive for COVID-19 without ever having to enter the patient's room. ${ }^{13}$ Remote monitoring of inpatients in the intensive care unit also allowed hospitals to conserve PPE with less frequent trips to isolation rooms that would have required new PPE with each new entrance. ${ }^{2}$ Elective procedures and appointments were postponed so that staff could focus on the influx of patients resulting from the virus and reduce the risk of exposure to healthy patients. The number of hospital staff working in person was reduced to decrease potential spread of the virus and forced clinicians to find a new way to provide care. ${ }^{6}$ At University Rey Juan Carlos (Madrid, Spain), telemedicine was used to triage patients presenting to the emergency department in an effort to determine which patients could be cared for virtually to reduce traffic and preserve resources. ${ }^{14}$

Telemedicine was incorporated into inpatient care in many hospitals to balance the supply of clinical services with the increasing demands. The aim was to limit contact with potentially infected patients to reduce the spread of the virus, decreasing the risk of hospital staff becoming infected. The conversion to telemedicine within the inpatient setting allowed higher-risk hospital staff, whether immunocompromised or elderly, to work remotely and still manage the increased patient volume without compromising their own health. Videoconferencing applications were also used by hospitals to help patients in isolation to communicate with family and friends who were restricted from visiting. ${ }^{2,6}$

Another serious implication of restricting access to hospitals not often discussed was the training of upcoming medical professionals including nurses, doctors, and pharmacists. Many had their training postponed when there was an increased need for healthcare staff. Telemedicine has allowed these students to participate in training activities, such as patient rounding, without putting them in high-risk situations where they could be exposed to COVID-19.2 Telemedicine created an environment in which healthcare trainees were included in and learnt from the healthcare team without entering the hospital. ${ }^{2}$ 
Tongji Hospital (Wuhan, China) used telemedicine services to supplement their typical inpatient workflow using a new system by monitoring patients with COVID-19 who were self-quarantined at home. As hospitals across the world encouraged patients with mild illness to stay home and self-quarantine rather than flood the emergency departments, this strategy was not without risks of its own. The condition of these patients could change rapidly, and the patient's condition could quickly develop into a critical situation, which would require inpatient care. Self-quarantine of patients delayed time to proper care and led to potential adverse patient outcomes. One team from Tongji Hospital conducted a retrospective study to evaluate patient outcomes when self-quarantined patients were monitored from home using telemedicine. Between $6^{\text {th }}$ January and $31^{\text {st }}$ January 2020 , patients suspected of having COVID-19 were given an initial questionnaire to determine if their case required inpatient care or could be monitored at home in self-quarantine. If the patient was deemed eligible for self-quarantine, they were set up with the electronic counselling system. The system allowed for two-way communication between the patient and an interdisciplinary team with two physicians, three nurses, a rehabilitation physician, and a psychologist. The patient updated their condition daily by answering questions regarding their health on an electronic counselling app on their phone. The health team then assessed the patient's temperature, heart rate, and oxygen saturation and provided feedback to the patient. At the end of the study, 74 patients who were diagnosed with COVID-19 had been eligible for self-quarantine. Sixty-eight participants effectively recovered during their self-quarantine without requiring inpatient care; however, six of the 74 patients had a worsening of their status and required hospital admission. All six patients were eventually discharged, and no deaths were reported during this study. With the help of telemedicine, 74 patients diagnosed with COVID-19 were effectively treated by only seven healthcare workers without any risk of transmission to hospital staff, other patients, or the general public..$^{15}$

In contrast to the implementation of telemedicine in the inpatient setting, outpatient settings became the most common use of telemedicine prepandemic and continue to serve a vital role as the pandemic continues. Conversion of outpatient visits to telemedicine allowed patients to continue care of their chronic conditions while not having to travel to clinic and risk exposure. The deferment of care of these chronic conditions caused by fear from the providers or the patients could have led to future health complications. It was essential that a patient's chronic conditions continued to be monitored and cared for in a timely fashion to prevent potential decline in their condition as a result of COVID-19.2 Prior to the pandemic, Duke University Health System (Durham, North Carolina, USA) had telemedicine practices in place; however, they were not widely used. Over a 4-week period (12 ${ }^{\text {th }}$ March-9 $9^{\text {th }}$ April 2020), Duke University Health System's telehealth visits increased from $<1 \%$ of total visits to $70 \%$ of total outpatient visits. At the peak of the pandemic in April, the Duke University Health System performed over 1,000 telehealth visits per day via phone and videoconferencing. ${ }^{2}$

New York University (NYU) Langone Health (New York City, New York, USA), was another institution that used telemedicine prior to the pandemic; however, not in a widespread manner. Prior to the coronavirus outbreak, telemedicine was only used in approximately 25 of over 500 ambulatory clinics in NYU, with fewer than 100 telehealth visits per day. On 19 ${ }^{\text {th }}$ March 2020, NYU Langone Health expanded telemedicine to all of its ambulatory clinic locations. Within 10 days, more than 7,000 telemedicine visits were performed, accounting for $>70 \%$ of the total ambulatory visit volume. Patient satisfaction remained unchanged throughout this transition based on surveys administered to the patients. ${ }^{10}$

West Tennessee Health (Jackson, Tennessee, USA), contrastingly, had plans to implement telemedicine practices prior to the pandemic; yet, they had not performed a single telemedicine visit. The COVID-19 outbreak pushed West Tennessee Health to rapidly carry out their telemedicine plans to keep healthy individuals out of waiting rooms. Throughout March 2020, the health system had a 1,300\% increase in telemedicine visits conducted throughout the month. ${ }^{16}$

Italy was one of the first European countries to shut down in response to the rapid increase in COVID-19 cases. In some Italian cities, a complete lockdown was implemented leading to a drastic 
decrease in the number of patients attending outpatient appointments. The Outpatient Rehabilitation Institute at IRCCS Istituto Ortopedico Galeazzi, Milan, Italy, transitioned all face-to-face visits to telemedicine visits on $16^{\text {th }}$ March $2020 . .^{17}$ A face-to-face visit was only deemed necessary, if at all, after a telemedicine consultation was performed. Within a few days, the institute developed emergency protocols to make the swift transition. Between $16^{\text {th }}$ March and $3^{\text {rd }}$ April 2020, the Rehabilitation Institute conducted 1,207 telemedicine outpatient visits. Only one out of every 200 patients were required to complete a face-to-face appointment following a telemedicine consultation, attributed to the inability to effectively care for the patient via telehealth. ${ }^{17}$ While the Rehabilitation Institute fully transitioned to telemedicine visits, provider satisfaction surveys were distributed to assess the efficacy of the transition. Mean satisfaction of telemedicine visits was 2.8 out of 3 and physicians indicated that they were "overall happy" with their experience using telemedicine. Even physicians who were not comfortable with technology prior to the transition reported being surprised with the high level of satisfaction they had while using telemedicine for outpatient visits. ${ }^{14}$

\section{FUTURE}

With the seemingly successful conversion to telemedicine, the question remains: How were the barriers to telehealth overcome so rapidly, and what does this mean for the future? One of the biggest barriers to telemedicine prepandemic was insurance regulations and lack of reimbursement for services. During the pandemic, telemedicine regulations were relaxed, and most telemedicine services were covered. Many insurance companies implemented temporary approval of telehealth services, which led to increased use., 610 In the USA, 'Medicare' patients were offered the same telemedicine coverage as the Medicare 'Advantage' patients. There was also an increase in state and federal government funding in an effort to promote telehealth in low-income areas in addition to the provision of necessary technology to complete remote visits. ${ }^{6}$ Perhaps the biggest change came on $17^{\text {th }}$ March 2020 , when the Office of Civil Rights, U.S. Department of Health and Human Services, announced that potential
Health Insurance Portability and Accountability Act (HIPAA) violations for using everyday communication technology for patient care would be waived. ${ }^{16}$ This vastly increased the virtual platforms that clinicians were able to use to provide patient care.

As a healthcare system, many lessons have been learned since the start of the COVID-19 outbreak. One of the biggest accomplishments realised from the pandemic is that many outpatient visits can be effectively managed via telemedicine without compromising patient care. ${ }^{7}$ The pandemic forced the use of telemedicine allowing patients, providers, and healthcare systems to appreciate the advantages of telemedicine, which included optimising patient care virtually and using it as a means to provide nonurgent follow-up visits. In many instances, the wireless connectivity and technology capabilities were already in place, it was just a matter of maximising their use. The COVID-19 pandemic has demonstrated that healthcare staff can quickly adapt to the new technologies needed to implement telemedicine. ${ }^{7,8,16}$ In the case of Duke University Health System, the hospital-wide use of telemedicine was started in mid-March and by $1^{\text {st }}$ May 2020 , staff, physicians, nurses, care specialists, pharmacists, and other healthcare professionals had been fully trained and were able to provide telemedicine in both the inpatient and outpatient setting. ${ }^{2}$

Previously, telemedicine was typically only adopted for specialised care; however, in light of COVID-19, healthcare workers have been able to recognise that telemedicine can also be utilised for routine patient care. ${ }^{2}$ Moving forward from the global pandemic, further implementation of telemedicine into everyday practice will require switching from crisis mode to sustainability. Ensuring that providers and healthcare workers are fully trained in telemedicine is essential. Healthcare providers are still required to follow both institution protocols and best practice guidelines to provide optimal patient care. No matter what platform a healthcare provider is using to treat a patient, they should always be practising at the top of their licence and providing the same standard of care to all patients whether face-to-face or virtually. Proper documentation and follow-up are still essential in the practice of telemedicine and the safety of the patient should never be jeopardised. ${ }^{7}$ It is also important to minimise the risk of physicians 
abusing the telemedicine system solely as a way to increase revenue. ${ }^{7}$ Other things to consider when moving forward with telemedicine is the ability of low-income or elderly individuals to access the device or network that is required for the use of telemedicine. Telemedicine is available to increase access to care. It should not be seen as a barrier that would prevent patients from accessing proper medical care. ${ }^{6}$ In addition to ensuring adequate access to necessary technology and resources for telemedicine, insurance coverage for telemedicine services is essential postpandemic."1

\section{CONCLUSION}

While the COVID-19 pandemic produced a feeling of mass chaos in many aspects of life, one positive impact of the pandemic has been the widespread implementation of telemedicine. Without the push from the pandemic, many health institutes would have continued to avoid the widespread implementation of telemedicine and may never have come to realise all the benefits telemedicine has to offer.

\section{References}

1. American Medical Association Telehealth implementation playbook. 2019. Available at: https://www.amaassn.org/system/files/2020-04/amatelehealth-implementation-playbook. pdf. Last accessed: 25 September 2020

2. Wosik $\mathrm{J}$ et al. Telehealth transformation: COVID-19 and the rise of virtual care. J Am Med Inform Assoc. 2020;27(6):957-62.

3. Barnett $\mathrm{ML}$ et al. Trends in telemedicine use in a large commercially insured population, 2005-2017. JAMA. 2018;320(20):21479.

4. Martin RD. Leveraging telecommuting pharmacists in the post-COVID-19 world. J Am Pharm Assoc. 2020;60(6):E113-5.

5. American Hospital Association. Fact sheet: Telehealth. 2019. Available at: https://www.aha.org/factsheet/ telehealth. Last accessed: 25 September 2020.

6. Contreras CM et al. Telemedicine: patient-provider clinical engagement during the COVID-19 pandemic and beyond. J Gastrointest Surg 2020;24(7):1692-7.

7. Bashshur $\mathrm{R}$ et al. Telemedicine and the COVID-19 pandemic, lessons for the future. Telemed J E Health. 2020;26(5):571-3.

8. Smith $A C$ et al. Telehealth for global emergencies: implications for coronavirus disease 2019 (COVID-19). J Telemed Telecare. 2020;26(5):30913.

9. Raposo VL. Telemedicine: the legal framework (or the lack of it) in Europe. GMS Health Technol Assess. 2016;12:Doc03.

10. Mann DM et al. COVID-19 transforms health care through telemedicine: evidence from the field. J Am Med Inform Assoc. 2020;27(7):1132-5.

11. Centers for Disease Control and Prevention. 2020. Using Telehealth Services. Available at: https://www. cdc.gov/coronavirus/2019-ncov/hcp/ telehealth.html. Last accessed: 25 September 2020.

12. Hong $Z$ et al. Telemedicine during the COVID-19 pandemic: experiences from Western China. J Med Internet Res. 2020;22(5);e19577.

13. Chou $\mathrm{E}$ et al. Onsite telemedicine strategy for coronavirus (COVID-19) screening to limit exposure in ED. Emerg Med J. 2020;37(6):335-7.

14. Barba R et al. Managing people, roles, and resources during COVID-19 surge. NEJM Catal Innov Care Deliv. 2020:10.1056/CAT.20.0152.

15. $\mathrm{Xu} \mathrm{H}$ et al. Monitoring and management of home-quarantined patients with COVID-19 using a WeChat-based telemedicine system: retrospective cohort study. J Med Internet Res. 2020;22(7):e19514.

16. Pirtle CJ et al. Implementation and optimization of telehealth during the COVID-19 public health crisis - our journey. Telehealth and Medicine Today. 2020;5(3).

17. Negrini $S$ et al. Feasibility and acceptability of telemedicine to substitute outpatient rehabilitation services in the COVID-19 emergency in Italy: an observational everyday clinical-life study. Arch Phys Med Rehabil. 2020;101(11):2027-32. 